\title{
Recent Advances in Stem Cell Researches and Their Future Perspectives in Regenerative Medicine
}

\author{
Youngsook Son ${ }^{1,2}$
}

Received: 7 November 2017/Accepted: 8 November 2017/Published online: 17 November 2017

(C) The Korean Tissue Engineering and Regenerative Medicine Society and Springer Science+Business Media B.V., part of Springer Nature 2017

A variety of stem cells with different origins and differentiation potency are expected to cure numerous degenerative diseases, chronic diseases and acute tissue injuries. Recent advances in stem cell biology and stem cell engineering provide new avenue for pluripotent stem cells (PSC), including embryonic stem cells (ESC), induced pluripotent stem cells (iPSC), and directly converted stemlike cells, which are essential resources for disease modeling, drug screening, and cell therapeutics for regenerative medicine. Also, stem cells derived from cord blood, amnionic membrane, and discarded tissues from surgical procedure are good resources for therapeutic cells, several of them have been clinically tried, and beneficial effects are obtained. On the other hand, endogenous stem cells with less differentiation potency than PSCs, also called as adult stem cells or tissue stem cells, are reserved in almost all tissues in our body and continuously supplying differentiated cells for the maintenance of tissues or organ function in our life time. Those adult stem cells are ex vivo cultured for expansion of therapeutic cells as patients' own autologous cell sources or allogeneic cell sources under GMP facility.

Youngsook Son

ysson@khu.ac.kr

1 Department of Genetic Engineering, College of Life Science and Graduate School of Biotechnology, Kyung Hee

University, 1732 Deogyeong-daero, Giheung-gu, Yongin-si, Gyeonggi-do 17104, Republic of Korea

2 Kyung Hee Institute of Regenerative Medicine, Kyung Hee University Hospital, 24 Kyungheedae-ro, Dongdaemun-gu, Seoul 02453, Republic of Korea
Recent clinical trials, mostly from adult stem cells and cord blood cells, strongly support their clinical benefits. Even though high medical expenses are payed comparing to conventional medication, efficacies are mostly claimed as paracrine effects of cell therapeutics instead of reparative stem cells remaining as tissue components in the repaired tissue. Therefore, scientific and technical innovations such as fate control of PSC, ex vivo cell expansion, safety issue, host engraftment, tissue repair, and development of proper protocol for clinical trials, are expected to solve current limitations and hurdles for successful stem cell therapeutics.

In this special issue, four reviews are invited to overview most important current critical issues. They provide new concepts and paradigm in the fields of stem cells and their application in regenerative medicine, especially in epigenetic regulation of reprogrammed pluripotent stem cells [1], secretomes [2] and exosomes [3], endogenous stem cells in homeostasis as well as in aging process [4], and propose their future perspectives at the end.

The first one overviews epigenetic dysregulation in pluripotency such as imprinting status and X-chromosome dosage compensation, mainly focusing $\mathrm{X}$-chromosome regulation in PSCs by Patterson et al. at Yale University, titled on "New Advances in Human X-chromosome Status from a Development and Stem Cell Biology". The second one overviews current status of stem cells therapy in liver disease and propose paracrine effects of transplanted cells as a new paradigm for the cure of liver disease by Kim et al. at Korea University, titled on "Current Understanding of Stem Cell and Secretome Therapies in Liver Disease". The third overviews a new concept of extracellular vesicles (EVs), so called exosome, loaded with proteins, mRNAs, noncoding RNAs, and membrane bounds lipid and their 
pathological implications in neurodegenerative disorders such as Parkinson's disease, Alzheimers' disease, and amyotrophic lateral sclerosis, and propose EVs as drug delivery model vehicles by Lee et al. at Catholic Kwandong University, titled on "Extracellular Vesicles in Neurodegenerative Disease; A double-edged Sword". Finally, the fourth one overviews current concept of endogenous stem cells in homeostasis and in the aging based on cell autonomous alterations, extrinsic environment, and stem cell trafficking expecting possible pharmacological targets for anti-aging and rejuvenation by Lim et al. at Kyung Hee University, titled on "Endogenous Stem Cells in Homeostasis and Aging".

This special issue will provide current status and future perspectives of stem cell researches and their translation to the clinic to TERM readers as well as new comer in this field and propose new concepts and ideas for scientific and technical advancement. Even though whole issues in stem cells and regenerative medicine are not covered in this special issue, most important progress, critical issue, new concepts in the stem cells, especially at the aspect of regenerative medicine, are fairly well summarized and discussed. These reviews may help to grasp fast development in stem cell biology and regenerative medicine and further escalate scientific and technological advancement.

Acknowledgement Editor of this special issue would like to express the deepest gratitude to all invited authors to make their valuable effort for this review.

\section{References}

1. Hoffman LM, Hall L, Batten JL, Young H, Pardasani D, Baetge $\mathrm{EE}$, et al. X-inactivation status varies in human embryonic stem cell lines. Stem Cells. 2005;23:1468-78.

2. Park SR, Kim JW, Jun HS, Roh JY, Lee HY, Hong IS. Stem cell secretome and its effect on cellular mechanisms relevant to wound healing. Mol Ther. 2017. https://doi.org/10.1016/j.ymthe.2017.09. 023.

3. Thompson AG, Gray E, Heman-Ackah SM, Mäger I, Talbot K, Andaloussi SE, et al. Extracellular vesicles in neurodegenerative disease-pathogenesis to biomarkers. Nat Rev Neurol. 2016;12: 346-57.

4. Oh J, Lee YD, Wagers AJ. Stem cell aging: mechanisms, regulators and therapeutic opportunities. Nat Med. 2014;20: 870-80. 